\title{
Investigation of an outbreak of mycobacteriosis in pigs
}

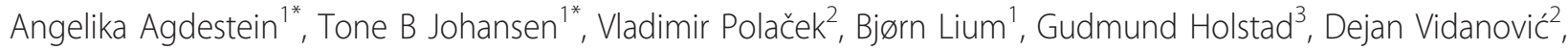
Sanja Aleksić-Kovačević ${ }^{4}$, Anne Jørgensen ${ }^{5}$, Jonas Žultauskas ${ }^{6}$, Sigrun F Nilsen ${ }^{1}$ and Berit Djønne ${ }^{1}$

\begin{abstract}
Background: A high proportion of pigs imported to Serbia from a Lithuanian breeding herd reacted positively against avian and/or bovine tuberculin. The pigs were euthanized and lesions characteristic for mycobacterial infection were detected. An investigation of potential mycobacteriosis in the pigs imported to Serbia and the possible source of infection in the Lithuanian herd were therefore initialised.
\end{abstract}

Results: Formalin fixed, paraffin embedded lymph nodes from tuberculin positive animals were examined by realtime PCR for IS1245 and IS6110. IS1245 was detected in 55\% and IS6110 in 11\% of the samples. Seven of the ten IS6110 positive samples were positive for IS1245. Eleven lymph nodes from 10 pigs and 15 environmental samples were collected from the Lithuanian breeding herd and cultured for mycobacteria. M. avium subsp. hominissuis was detected in all lymph nodes and from eight samples of peat and sawdust. Isolates with identical and related IS1245- and IS1311 RFLP profiles were detected from swine and peat.

Conclusions: This study demonstrated cross reactions between avian and bovine tuberculin in pigs. Real-time PCR indicated infection with M. avium in the Serbian pigs. However, as a small proportion of the lymph nodes were positive for IS6110, infection with bacteria in the M. tuberculosis complex could not be ruled out. Analyses confirmed the presence of M. avium subsp. hominissuis in porcine and environmental samples from the Lithuanian breeding herd. The results indicate peat as a source of M. avium subsp. hominissuis infection in these pigs, and that the pigs imported to Serbia were infected with M. avium subsp. hominissuis.

\section{Background}

Mycobacterium avium, belonging to the Mycobacterium avium complex (MAC), has been divided into the subspecies avium, paratuberculosis and silvaticum [1]. More recently, M. avium subsp. avium has been further divided into M. avium subsp. avium and M. avium subsp. hominissuis [2]. M. avium has been isolated from different sources, such as water, pools, soil, plants and bedding material [3]. M. avium subsp. avium mainly cause generalised tuberculosis in poultry and wild birds, while $M$. avium subsp. hominissuis is an opportunistic pathogen, infecting mainly swine and humans $[2,4,5]$. M. bovis is the main agent causing tuberculosis in cattle, while M. tuberculosis primarily causes tuberculosis in humans. Both belong to the M. tuberculosis complex (MTC) and

\footnotetext{
* Correspondence: angelika.agdestein@vetinst.no; tone.bjordaljohansen@vetinst.no

${ }^{1}$ Norwegian Veterinary Institute, P. O. Box 750 dep., N-0106 Oslo, Norway Full list of author information is available at the end of the article
}

can lead to infections in pigs. Mycobacteriosis in pigs is mainly caused by $M$. avium subsp. hominissuis, and infection can lead to economic losses due to condemnation of carcasses. However, the MTC can cause similar lesions, and is a differential diagnosis with serious implications for the herd [6-8].

Intradermal tuberculin testing can be used for ante mortem diagnosis of tuberculosis in pigs $[4,9]$. The single intradermal comparative test entails simultaneous injection of bovine and avian tuberculin. Animals infected with MTC tend to show greater reaction to bovine tuberculin than to avian tuberculin, while animals exposed for $M$. avium or other mycobacteria tend to promote the reverse reaction. Cross reactions are seen, but although the sensitivity and the specificity of the test are imperfect, the test has an important function, especially in herd diagnosis $[9,10]$. Post mortem, mycobacterial infections can be diagnosed by pathological examination combined with histopathology. The histological diagnosis of tuberculosis
C Biomed Central

(ㄷ) 2011 Agdestein et al; licensee BioMed Central Ltd. This is an Open Access article distributed under the terms of the Creative Commons Attribution License (http://creativecommons.org/licenses/by/2.0), which permits unrestricted use, distribution, and reproduction in any medium, provided the original work is properly cited. 
comprises detection and localisation of acid fast bacilli in granulomatous lesions [11]. Culturing of mycobacteria is the gold standard for diagnosis, but molecular tests are also used $[4,12]$. Formalin fixed tissue can be used for analysis by PCR, and real time PCR is considered more sensitive than conventional PCR for such material [13]. Different genetic elements as the mobile insertion sequences (IS) can be used for diagnosis and genetic typing of mycobacteria. IS1245 is considered specific for $M$. avium [14] and IS6110 for the MTC [15], whereas IS901 is found only in M. avium subsp. avium [16].

In June 2007, 217 pigs were exported from a breeding herd in Lithuania to Serbia. All pigs were born between December 2006 and April 2007. During the quarantine period in Serbia, the pigs were skin tested with both avian and bovine tuberculin, as ordered by the Serbian Veterinary Directorate. Altogether 62 pigs reacted positively against avian and/or bovine tuberculin. One pig was euthanized, and lesions compatible with mycobacteriosis were revealed. According to instructions from the Serbian Veterinary Directorate, all tuberculin positive pigs were euthanized. The aim of the present study was to examine the remaining pigs imported to Serbia in order to determine whether they were infected by mycobacteria able to cause lesions and tuberculin reactions as observed. In addition, the possible source of infection in the Lithuanian breeding herd was investigated.

\section{Methods}

\section{Tuberculin testing and post mortem examinations of pigs} imported to Serbia

Tuberculin retesting of 154 out of the 155 pigs negative on the first examination was performed two months after the first test, a time interval regarded as sufficient to allow desensitation to wane [12]. Both avian and bovine tuberculin were used as indicated by the producer (Bioveta, Ivanovicena Hané, Czech Republic), followed by rigorous interpretation of results [12]. Thereafter, all remaining pigs were euthanized and examined for lesions compatible with tuberculosis, such as enlargement, miliar necrosis, caseous necrosis and calcification. Formalin fixation and paraffin embedding (FFPE) of lymph nodes from tuberculin positive pigs was performed as described [17]. The paraffin sections (3-5 $\mu \mathrm{m}$ thick) were stained with haematoxylin and eosin (HE) and Ziehl-Neelsen (ZN) staining for microscopic examination and detection of acid fast bacteria.

Samples from 25 randomly chosen tuberculin positive pigs with gross lesions characteristic for mycobacteriosis were sent for culturing of mycobacteria at the Department of Tuberculosis, Clinical Centre in Kraljevo, Serbia. Approximately 2 grams of tissue from mesenterial lymph nodes were grinded and decontaminated with $2 \% \mathrm{NaOH}$.
Isolation was performed on Löwenstein-Jensen medium with glycerol at $37^{\circ} \mathrm{C}$ for 8 weeks [12].

\section{Real-time PCR from FFPE lymph nodes from pigs imported to Serbia}

One hundred and fifteen FFPE lymph nodes from 100 pigs were sent to the Norwegian Veterinary Institute, and realtime PCRs for detection of DNA sequences of M. avium and MTC were performed. For extraction of DNA Nucleon HT (Tepnel Life Sciences, Manchester, UK) was applied, following the protocol provided by the manufacturer. From mycobacterial isolates and non-fixed tissue samples, DNA was extracted by Nuclisens ${ }^{\circledR}$ easyMag ${ }^{\circledR}$ (BioMérieux, Inc., Durham, NC, USA) following the manufacturer's instructions. Three singleplex real-time PCRs were developed, for IS1245 and IS6110 specific for M. avium and MTC respectively, and for the porcine $\beta$ globin gene. Amplification of the latter sequence was used as a positive control for successful DNA extraction from the samples. Primers and 6FAM labelled TaqMan MGB probes were designed using the program Primer 3.0 http://frodo.wi.mit.edu/primer3/ (Table 1). Real-time PCR was performed using Stratagene Mx3005P (Stratagene, La Jolla, CA, USA). Reaction mixtures had a total volume of $20 \mu \mathrm{l}$, consisting of $2 \mu \mathrm{l}$ template DNA at a concentration of 10-15 ng/ $\mu \mathrm{l}, 10 \mu \mathrm{l}$ PerfeC Ta ${ }^{\circledR}$ qPCR FastMix ${ }^{\circledR}$ (Quanta Biosciences, Gaithersburg, MD, USA), and primers and probes at a final concentration of 400 and $150 \mathrm{nM}$, respectively.

DNA extracted from FFPE lymph nodes from animals clinically infected with $M$. avium subsp. hominissuis or M. bovis, verified by culture prior to fixation, were used for optimisation of PCR conditions. DNA extracted from M. avium ATCC 25291, M. bovis BCG (Danish strain 1331) and from a porcine spleen, were used as positive controls for IS1245, IS6110 and $\beta$-globin. Ultrapure Milli-Q water was used for adjustment of volume and concentrations and as a template substitute in negative controls.

The real-time PCR reactions were initiated with denaturation at $95^{\circ} \mathrm{C}$ for $10 \mathrm{~min}$, followed by 45 runs of the following thermal cycle: $95^{\circ} \mathrm{C}$ for $3 \mathrm{sec}$ and $60^{\circ} \mathrm{C}$ for $30 \mathrm{sec}$. All samples and controls were run as single measurements. The results were analysed using Stratagene MxPro 4.10 software (Stratagene), applying the automatic calculation of the threshold fluorescence. Ct values above 40 were regarded as negative as recommended by the manufacturer (Stratagene).

\section{Post mortem examinations of animals and examinations} of environmental samples from the Lithuanian herd Six cervical and five mesenterial lymph nodes from ten randomly selected, clinically healthy pigs from the original 
Table 1 Primers and probes used in this study

\begin{tabular}{llll}
\hline Target & Size of amplified sequence $(\mathbf{b p})$ & Primers and probes & GenBank accession no. \\
\hline IS1245 & 82 & Primer 41: ggtgagcggatcactcaag* & L33879 \\
& & $\begin{array}{l}\text { Primer 116: ggagaagcccgatgaac } \\
\text { Probe 3: caagccttgatcgacgcgga }\end{array}$ & \\
\hline IS6110 & 101 & Primer 149: gccaactacggtgtttacgg & X17348.1 \\
& & $\begin{array}{l}\text { Primer 150: agttggtcatcagccgttc } \\
\text { Probe 6: gggcatcgaggtggccagat }\end{array}$ & \\
\hline Porcine $\beta$-globin & 119 & Primer 120: gggggttgcaatttattcct & X86791 \\
gene & & Primer 121: tgaatcacggtcctgtgaaa & \\
& & Probe 4: cgcagattcccaaccttcgc & \\
& & &
\end{tabular}

Lithuanian herd were collected at slaughter for gross pathology, culturing and typing of mycobacteria. Gross pathology of the lymph nodes was evaluated upon incision. Thereafter, culturing of the samples was performed as described previously $[18,19]$. Briefly, approximately two grams of tissue from lymph nodes or internal organs were homogenised, decontaminated with $5 \%$ oxalic acid and inoculated on different media with and without pyruvate and incubated at $37^{\circ} \mathrm{C}$ for up to two months [18].

Samples from the herd facilities; four from tap water, six from peat, four from sawdust and one scraping from the inside of a water pipe were analysed. The samples of peat and sawdust were taken from storage facilities, and had not been in contact with the pigs before sampling. All samples are described in Table 2. Pre-treatment of the environmental samples was performed slightly different depending on the type of material. Samples from peat and sawdust were soaked in an equal amount of sterile distilled water for $24 \mathrm{~h}$. Water samples were centrifuged at $3500 \mathrm{rpm}$ for 15 minutes and the pellets were processed further. For all environmental samples, decontamination was performed by $4 \% \mathrm{NaOH}$ followed by $5 \%$ oxalic acid with $0.1 \%$ malachite green, otherwise culturing was performed as described above. $\mathrm{ZN}$ positive isolates were identified by AccuProbe Mycobacterium avium culture identification test ${ }^{\mathbb{B}}$ (GenProbe Inc., San Diego, CA, USA). All isolates were examined for the presence of IS901 by PCR using $1 \mathrm{U}$ AmpliTaq ${ }^{\circledR}$ DNA

Table 2 Samples from the Lithuanian pig herd cultured for mycobacteria

\begin{tabular}{lll}
\hline Samples & No. examined & No. positive on culture \\
\hline Lymph node, cervical* $^{*}$ & 6 & 6 \\
Lymph node, mesenterial* $^{*}$ & 5 & 5 \\
Peat & 6 & 6 \\
Sawdust & 4 & 2 \\
Tap water & 4 & 0 \\
Water pipe & 1 & 0 \\
\hline
\end{tabular}

*Ten pigs were sampled, two isolates originated from different lymph nodes in the same animal polymerase (Applied Biosystems, Foster City, CA, USA). Primers 901a and 901c were used for the amplification of IS901 [20]. PCR conditions were as described earlier [18]. The reference strain $M$. avium subsp. avium ATCC25291 was used as a positive control, and Milli-Q water as negative control.

\section{Restriction Fragment Length Polymorphism (RFLP) on isolates from the Lithuanian herd}

IS1311- and IS1245 RFLP were performed on all isolates as described previously $[18,21,22]$. The avian reference strain ATTC25291 was used as a positive control and run on each gel [22]. The probes used for IS1245 and IS1311 RFLP were described by Johansen et al. $[18,21]$. The resulting RFLP patterns were analysed by visual inspection and by using the BioNumerics software (version 4.0, Applied Maths, Kortrijk, Belgium) as described previously [18]. Optimization and tolerance were set to $1.5 \%$ for IS1311 RFLP and to $0.8 \%$ for IS1245 RFLP. With these settings, the reference strain clustered with $100 \%$ similarity. A final dendrogram of the composite dataset of the two RFLP experiments was calculated using the similarity by the average from experiments, and the UPGMA clustering method. Correction for internal weights was used. The similarity cut-off was set at $80 \%$ to define clusters [18].

\section{Results}

Tuberculin testing and post mortem examinations of pigs imported to Serbia

Altogether 135 pigs showed positive tuberculin reactions on retesting of the pigs; 130 strong and two weak positive on avian and 77 strong and three weak positive on bovine tuberculin. Seventy-seven animals were positive on both avian and bovine tuberculin, 55 only on avian and three only on bovine. Only 19 of the tested animals showed negative reactions to both avian and bovine tuberculin. Details on reaction patterns against the two types of tuberculin are listed for 92 of the animals in Table 3.

Post mortem examination was performed on 100 pigs. Granulomatous lesions compatible with mycobacterial infection were found in mesenterial lymph nodes from 
Table 3 Results from real time PCR and tuberculin testing of 92 tuberculin positive Serbian pigs

\begin{tabular}{|c|c|c|c|c|}
\hline IS6110 PCR & IS1245 PCR & Bovine tuberculin & Avian tuberculin & No. of animals \\
\hline+ & + & + & + & 3 \\
\hline+ & + & - & + & 4 \\
\hline+ & - & + & + & 2 \\
\hline+ & - & - & + & 1 \\
\hline- & + & + & + & 22 \\
\hline- & + & - & + & $20^{a}$ \\
\hline- & + & + & - & 2 \\
\hline- & - & + & - & 1 \\
\hline- & - & + & + & $21^{\mathrm{b}}$ \\
\hline- & - & - & + & $16^{\mathrm{c}}$ \\
\hline
\end{tabular}

ane sample had no pathological lesions

${ }^{\mathrm{b}}$ Six samples had no pathological lesions

'One sample had no pathological lesions

88 pigs. Macroscopic lesions were detected from 43 pigs, and only microscopic lesions from 45 pigs. Similar lesions were detected in the ovaries of some sows. In 12 pigs, no lesions were detected. The granulomas were composed of focal accumulation of inflammatory cells in which macrophages, epithelioid cells, multinucleated giant cells and lymphocytes predominated. Caseous necrotic areas were present in some granuloma. Numerous eosinophils and myofibroblasts were present within the granuloma. $\mathrm{ZN}$ positive rods were detected in five of the samples. Unfortunately, no growth of mycobacteria was detected in the samples sent for culturing.

\section{Real-time PCR from FFPE lymph nodes from pigs imported to Serbia}

The Norwegian Veterinary Institute received 115 FFPE lymph nodes from the Serbian pigs. Twenty-three samples were excluded from real-time PCR analysis. Only one sample was included from each pig. Additionally, samples with uncertain sample ID or poor DNA quality measured by negative result on real-time PCR for the porcine $\beta$-globin gene were excluded. Of the 92 remaining samples analysed for the presence of IS1245 by realtime PCR, 51 (55.4\%) were regarded positive. Ten $(10.9 \%)$ of the 92 samples analysed by IS6110 real-time PCR were considered positive, all but three were however simultaneously positive in IS1245 PCR. The PCR products of the samples positive for IS6110 were additionally sequenced and compared to published sequences by NCBI BLAST http://blast.ncbi.nlm.nih.gov/Blast.cgi to confirm their identity. Of the pigs with neither microscopic nor macroscopic lesions, only one of eight samples examined was positive for IS1245 by PCR, and none were positive for IS6110. In samples from 38 of the tuberculin reactors no IS element could be detected. Responses seen in connection with tuberculin results are given in Table 3.
Post mortem examinations of animals and examinations of environmental samples from the Lithuanian herd The lymph nodes collected from clinically healthy pigs in Lithuania were not enlarged and had no external lesions, but on incision, yellowish-white caseous foci of a few millimetres in diameter were revealed on the cut surface of all lymph nodes. M. avium was isolated from all lymph nodes examined from the Lithuanian herd, from all samples of peat and from two of the sawdust samples. From the remaining environmental samples, including the water samples, no mycobacteria were detected (Table 2). None of the isolates harboured IS901, and they were therefore assigned as M. avium subsp. hominissuis.

\section{Restriction Fragment Length Polymorphism (RFLP)} analysis of the isolates from the Lithuanian herd

The RFLPs detected between two and eight copies of IS1311 and between 11 and 21 copies of IS1245 in the isolates. As shown previously [18], the IS1245 RFLP showed a higher discriminative power than the IS1311 RFLP. Discrimination of the isolates was improved by a concomitant use of IS1311 and IS1245 RFLP.

Three clusters, where M. avium subsp. hominissuis isolates shared a similarity of more than $80 \%$, were named A-C (Figure 1). One isolate from peat did not belong to any cluster. Cluster A was composed of six porcine isolates and one from peat, and cluster $B$ consisted of nine isolates; five porcine and four from peat. One isolate from peat (\#1979) was identical to two porcine isolates (\#1967 and \# 1974). The two isolates originating from sawdust belonged to cluster $C$. They showed identical RFLP profiles that were different from the porcine isolates. Isolate \#1966 and \#1976 originated from the same pig, but clustered in different clusters.

Eight isolates had identical profiles by IS1311 RFLP; four porcine (\#1967, \#1969, \#1974 and \#1976) and four 


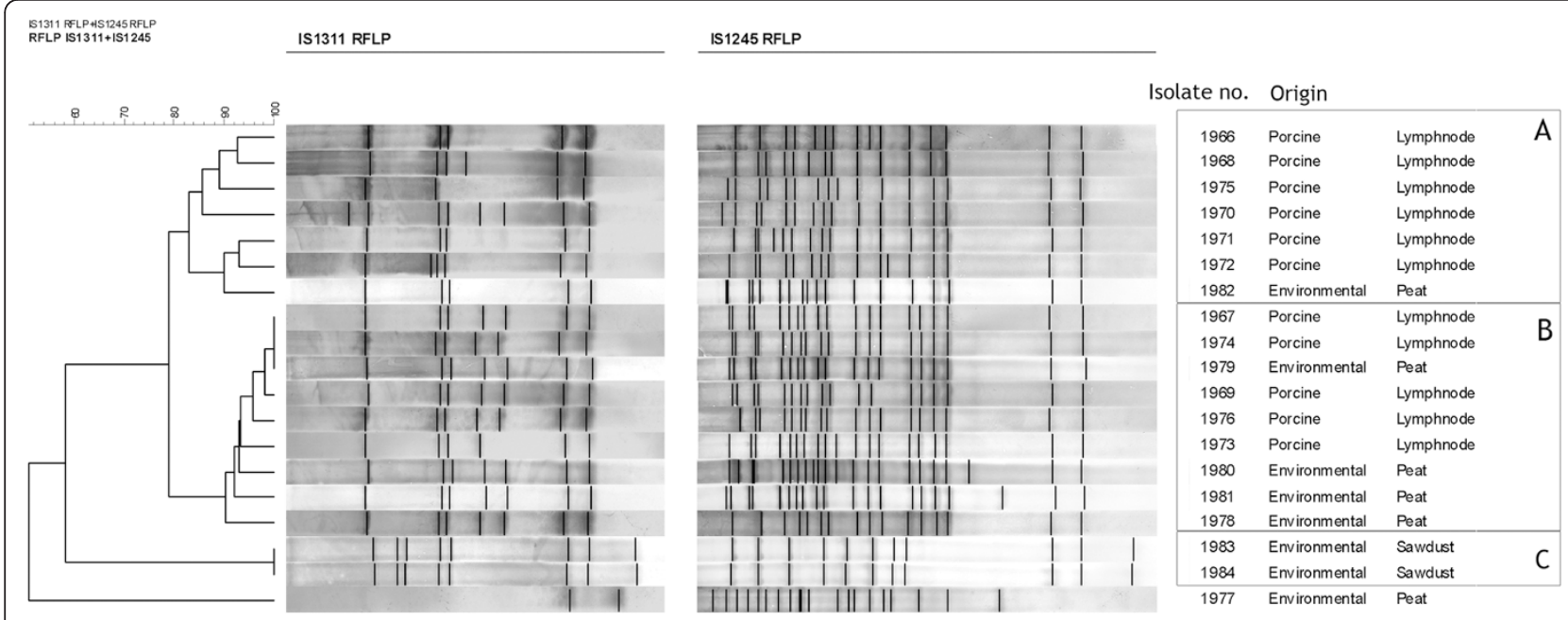

Figure 1 Cluster analysis based on RFLP analysis of isolates of Mycobacterium avium subspecies hominissuis. The dendrogram was calculated by using the similarity by the average from experiments and the unweighted-pair group method using arithmetic averages clustering method. Correction for internal weights was used. Two probes were used for RFLP; IS1311 and IS1245. Identification number and origin of the isolates are listed in columns on the right. Clusters based on $80 \%$ similarity are indicated with frames and marked A, B, and C.

peat isolates (\# 1978, \#1979, \#1980 and \#1981). In the IS1245 RFLP, however, only three of these isolates showed identical profiles (\#1967, \#1974 and \#1979). The other five isolates showed differences of one to three bands compared to the profile of the three identical isolates (Figure 1).

\section{Discussion}

Tuberculin reactions and pathological lesions in the pigs imported to Serbia indicated mycobacterial infection. More than $50 \%$ of the animals reacted positive for both avian and bovine tuberculin. Only three pigs reacted positive to bovine and negative to avian tuberculin. None of the FFPE lymph nodes originating from these three pigs were positive on real time PCR for IS6110, while two were positive for IS1245. Cross reactions between avian and bovine tuberculin are commonly observed in cattle [10], and our results indicate that such reactions are common in pigs as well.

In all but 12 pigs, pathological lesions were detected, indicating a very high infection rate in the herd. Only one of the PCR examined FFPE lymph nodes from the pigs without pathological lesions was positive for IS1245 by PCR, confirming that a thorough macroscopic and microscopic examination will detect most of the infected pigs. However, neither tuberculin reactions nor pathology can differentiate between infections with $M$. bovis and M. avium.

Culturing was unsuccessful, possibly due to low number of bacteria in the samples, uneven distribution of bacteria in the lesions or the lack of different growth media used for culture. As the samples were discarded, reculturing was not possible. Still, positive reactions for IS1245 on real-time PCR confirmed presence of $M$. avium in 55\% of the FFPE lymph nodes. It was therefore assumed that the animals imported to Serbia were infected with $M$. avium.

Real-time PCR for IS6110 gave positive reactions in FFPE lymph nodes from ten pigs in Serbia. This might indicate an infection with the MTC, possibly before import. However, this is unlikely as Lithuania is officially free of bovine tuberculosis today and the last occurrence in domestic animals was reported in 2001 [23,24]. In Serbia, bovine tuberculosis is still present in domestic animals [23], but considering the relatively short lapse of time between import to Serbia and sampling of the lymph nodes, and the strict quarantine measures applied, it seems unlikely that the animals were infected with MTC after import. The results are possibly due to false positive reactions or to cross reactions with DNA sequences yet to be described. However, without the possibility to culture mycobacteria from the IS6110 positive lymph nodes, it is not possible to conclude with $100 \%$ certainty whether any of these pigs were infected by bacteria within the MTC.

The sensitivity and specificity of the real-time PCRs for IS6110 and IS1245 cannot be determined as culturing in Serbia was not successful for these samples. Real-time PCR is considered an efficient, highly sensitive and specific diagnostic tool, using the appropriate controls and protocols. However, false negatives on real-time PCR might be due to impaired DNA quality, as formalin fixation and paraffin embedding are reported to compromise the integrity of DNA [25]. To counteract the negative 
effect of formalin fixation and paraffin embedding on DNA quality, the target sequences were designed to be short ( $\leq 200 \mathrm{bp}$ ) and samples with $\mathrm{Ct}$ values up to 40 were considered positive. These measures lower the possibility of false negatives, but also increase the risk of false positive results. Seven of the ten samples positive for IS6110 were also positive for IS1245. This could indicate multiple infections or false positive reactions. The specificity of IS6110 PCR in clinical material have been described to be between $96.1 \%$ and $100 \%$ [26,27], but could be lower for real time PCR in FFPE lymph nodes.

The animals were kept in quarantine in Serbia, and the first tuberculin testing was performed only 10 days after arrival. It was therefore assumed that any mycobacterial infection was contracted in Lithuania before export. Through culture and RFLP analysis of samples from the Lithuanian breeding facility, this suspicion was further confirmed, as M. avium subsp. hominissuis was detected in several samples from pigs and from their environment. The lymph nodes sampled from the Lithuanian breeding herd were randomly collected from healthy swine at slaughter, showing no visible external lesions. Still, all were positive for M. avium subsp. hominissuis, indicating a very high level of infection in the herd. M. avium subsp. hominissuis was also isolated from half of the sawdust and all peat samples, showing that the pigs lived in a contaminated environment.

Identical and closely related isolates from peat and porcine lymph nodes were detected by RFLP analysis, indicating peat as a possible source of infection for the pigs. Four porcine and four peat isolates identical on IS1311 RFLP, showed minor differences in the IS1245 RFLP profiles. Such differences have earlier been observed both on subculture and in multiple cultures from the same individual $[28,29]$. Tenover et al. [30] demonstrated how one mutation could result in two or three band differences in pulsed field gel electrophoresis patterns and that such patterns could be considered subtypes of the outbreak pattern when isolates are epidemiologically linked. A similar interpretation may be valid for RFLP patterns, which would indicate a close genetic relationship between these eight isolates. The discrepancies in RFLP profiles between porcine isolates and isolates originating from sawdust indicate that mycobacteria in sawdust were not the source of infection in the pigs. Two isolates originating from the same animal clustered in two different clusters. This suggests an infection with two different variants, and could be related to a high infection pressure in the environment. Such infections have been described earlier, both in humans and in swine [31-34].

In the Lithuanian herd, iron enriched peat was fed to piglets as an iron supplement and for regulation of intestinal function, while sawdust was used for bedding. M. avium subsp. hominissuis has been isolated from both peat and sawdust used in swine farms earlier, and related RFLP types have been detected in both peat and swine and in sawdust and swine [35-37]. Feeding contaminated peat to small piglets could explain the high level of infection in this herd, because young animals are more susceptible to infectious agents than older animals. After the source of infection was identified, all peat and sawdust was removed, the facilities were cleaned and disinfected, and new heat treated peat was introduced in the herd.

\section{Conclusions}

Cross reaction between avian and bovine tuberculin was demonstrated in pigs imported from Lithuania to Serbia. Real time PCR analysis of FFPE lymph nodes from pigs positive on both tuberculins indicated infection with M. avium. However, a small proportion of FFPE samples were PCR positive for MTC. M. avium subsp. hominissuis was subsequently detected by culture from randomly selected porcine lymph nodes and from peat and sawdust from the Lithuanian herd facilities. Isolates from pigs and peat showed genetic relationship by RFLP, suggesting that the infection probably originated from contaminated peat and that the exported pigs were infected by $M$. avium subsp. hominissuis at an early age.

\section{Acknowledgements and funding}

The project was funded by the Norwegian Research Council, and the Norwegian Veterinary Institute. Norsvin International is acknowledged for cooperation. Vivi Myrann and Nina Fundingsrud are greatly acknowledged for their excellent technical assistance.

\section{Author details}

${ }^{1}$ Norwegian Veterinary Institute, P. O. Box 750 dep., N-0106 Oslo, Norway. 2Veterinary Specialized Institute "Kraljevo", Žička 34, 36000 Kraljevo, Serbia. ${ }^{3}$ Norwegian School of Veterinary Science, P. O. Box 8156 Dep., N-0033 Oslo, Norway. ${ }^{4}$ Department of Pathology, Faculty of Veterinary Medicine, Belgrade University, Bulevar Oslobođenja 18, 11000 Belgrade, Serbia. ${ }^{5}$ Norwegian Pig Health Service, Animalia, P.O. Box 396 Økern, N-0513 OSLO, Norway. ${ }^{6}$ Norsvin Lithuania, Müro Strèvininkai, 56202 Kaišiadoriųraj, Lithuania.

\section{Authors' contributions}

AA and TBJ contributed to conception and design, culture and molecular studies, data analyses and drafting of the manuscript. VP, SAK and DV contributed to tuberculin testing, pathological and histopathological analyses and writing of manuscript. AJ and JŽ contributed to collection of samples and writing of manuscript. SFN contributed to molecular studies and writing of manuscript. BL contributed to conception and design, pathological analyses and writing of the manuscript. GH and BKD contributed to conception and design, analysed data and helped to draft the manuscript. All authors have read and approved the final manuscript.

Received: 7 February 2011 Accepted: 21 October 2011

Published: 21 October 2011

\section{References}

1. Thorel MF, Krichevsky M, Levy-Frebault W: Numerical taxonomy of mycobactin-dependent mycobacteria, emended description of Mycobacterium avium, and description of Mycobacterium avium subsp. avium subsp. nov., Mycobacterium avium subsp. paratuberculosis subsp. nov., and Mycobacterium avium subsp. silvaticum subsp. nov. Int I Syst Bacteriol 1990, 40:254-260. 
2. Mijs W, de Haas P, Rossau R, van der Laan T, Rigouts L, Portaels F, van Soolingen D: Molecular evidence to support a proposal to reserve the designation Mycobacterium avium subsp. avium for bird-type isolates and ' $M$. avium subsp. hominissuis' for the human/porcine type of $M$. avium. Int J Syst Evol Microbiol 2002, 52:1505-1518.

3. Inderlied CB, Kemper CA, Bermudez LE: The Mycobacterium avium complex. Clin Microbiol Rev 1993, 6:266-310.

4. Thorel MF, Huchzermeyer HF, Michel AL: Mycobacterium avium and Mycobacterium intracellulare infection in mammals. Rev Sci Tech 2001, 20:204-218.

5. Turenne CY, Wallace R Jr, Behr MA: Mycobacterium avium in the postgenomic era. Clin Microbiol Rev 2007, 20:205-229.

6. Komijn RE, de Haas PE, Schneider MM, Eger T, Nieuwenhuijs JH, van den Hoek RJ, Bakker D, Zijd Erveld FG, van Soolingen D: Prevalence of Mycobacterium avium in slaughter pigs in The Netherlands and comparison of IS1245 restriction fragment length polymorphism patterns of porcine and human isolates. J Clin Microbiol 1999, 37:1254-1259.

7. Thorel MF, Huchzermeyer $\mathrm{H}$, Weiss R, Fontaine JJ: Mycobacterium avium infections in animals. Literature review. Vet Res 1997, 28:439-447.

8. Biet F, Boschiroli ML, Thorel MF, Guilloteau LA: Zoonotic aspects of Mycobacterium bovis and Mycobacterium avium-intracellulare complex (MAC). Vet Res 2005, 36:411-436.

9. Cvetnic Z, Spicic S, Benic M, Katalinic-Jankovic V, Pate M, Krt B, Ocepek M: Mycobacterial infection of pigs in Croatia. Acta Vet Hung 2007, 55:1-9.

10. Rua-Domenech R, Goodchild AT, Vordermeier HM, Hewinson RG, Christiansen $\mathrm{KH}$, Clifton-Hadley RS: Ante mortem diagnosis of tuberculosis in cattle: a review of the tuberculin tests, gamma-interferon assay and other ancillary diagnostic techniques. Res Vet Sci 2006, 81:190-210.

11. Ulrichs $T$, Lefmann $M$, Reich $M$, Morawietz $L$, Roth $A$, Brinkmann $V$, Kosmiadi GA, Seiler P, Aichele $P$, Hahn $H$, et al: Modified immunohistological staining allows detection of Ziehl-Neelsen-negative Mycobacterium tuberculosis organisms and their precise localization in human tissue. J Pathol 2005, 205:633-640.

12. OIE: Manual of diagnostic tests and vaccines for terrestrial animals. Bovine tuberculosis. The World Organisation for Animal Health (OIE); 2009 [http://www.oie.int/fileadmin/Home/eng/Health_standards/tahm/ 2.04.07_BOVINE_TB.pdf].

13. Gomez-Laguna J, Carrasco L, Ramis G, Quereda JJ, Gomez S, Pallares FJ: Use of real-time and classic polymerase chain reaction assays for the diagnosis of porcine tuberculosis in formalin-fixed, paraffin-embedded tissues. J Vet Diagn Invest 2010, 22:123-127.

14. Guerrero C, Bernasconi C, Burki D, Bodmer T, Telenti A: A novel insertion element from Mycobacterium avium, IS1245, is a specific target for analysis of strain relatedness. J Clin Microbiol 1995, 33:304-307.

15. Thierry D, Cave MD, Eisenach KD, Crawford JT, Bates JH, Gicquel B, Guesdon JL: IS6110, an IS-like element of Mycobacterium tuberculosis complex. Nucleic Acids Res 1990, 18:188.

16. Kunze ZM, Wall S, Appelberg R, Silva MT, Portaels F, McFadden JJ: IS901, a new member of a widespread class of atypical insertion sequences, is associated with pathogenicity in Mycobacterium avium. Mol Microbiol 1991, 5:2265-2272.

17. Polacek V, Prodanov J, Lazic S, Petrovic T, Rasic Z, Aleksic-Kovacevic S: Immunohistochemical detection of B and T lymphocytes in mandibular lymph nodes of experimentally infected piglets with classical swine fever virus. Acta Vet (Beograd) 2007, 57:199-208.

18. Johansen TB, Olsen I, Jensen MR, Dahle UR, Holstad G, Djønne B: New probes used for IS1245 and IS1311 restriction fragment length polymorphism of Mycobacterium avium subsp. avium and Mycobacterium avium subsp. hominissuis isolates of human and animal origin in Norway. BMC Microbiol 2007, 7:14

19. Valheim M, Djonne B, Heiene R, Caugant DA: Disseminated Mycobacterium celatum (type 3 ) infection in a domestic ferret (Mustela putorius furo). Vet Pathol 2001, 38:460-463.

20. Ahrens P, Giese SB, Klausen J, Inglis NF: Two markers, IS901-IS902 and p40, identified by PCR and by using monoclonal antibodies in Mycobacterium avium strains. J Clin Microbiol 1995, 33:1049-1053.

21. Johansen TB, Djønne B, Jensen MR, Olsen I: Distribution of IS1311 and IS1245 in Mycobacterium avium subspecies revisited. J Clin Microbiol 2005, 43:2500-2502.
22. van Soolingen D, Bauer J, Ritacco V, Leao SC, Pavlik I, Vincent V, Rastogi N Gori A, Bodmer T, Garzelli C, et al: IS1245 restriction fragment length polymorphism typing of Mycobacterium avium isolates: proposal for standardization. J Clin Microbiol 1998, 36:3051-3054.

23. WAHID: World Animal Health Information Database (WAHID) Interface. The World Organisation for Animal Health (OIE) 2010 [http://web.oie.int/ wahis/public.php?page=home].

24. Reviriego Gordejo FJ, Vermeersch JP: Towards eradication of bovine tuberculosis in the European Union. Vet Microbiol 2006, 112:101-109.

25. Barcelos D, Franco MF, Leao SC: Effects of tissue handling and processing steps on PCR for detection of Mycobacterium tuberculosis in formalinfixed paraffin-embedded samples. Rev Inst Med Trop Sao Paulo 2008, 50:321-326.

26. Ben K, Ben SW, Marzouk M, Ferjeni A, Ghezal S, Boukadida J: Evaluation of a simplified IS6110 PCR for the rapid diagnosis of Mycobacterium tuberculosis in an area with high tuberculosis incidence. Pathol Biol (Paris) 2011, 59:161-165.

27. Chawla K, Gupta S, Mukhopadhyay C, Rao PS, Bhat SS: PCR for M. tuberculosis in tissue samples. J Infect Dev Ctries 2009, 3:83-87.

28. Bauer J, Andersen AB: Stability of insertion sequence IS1245, a marker for differentiation of Mycobacterium avium strains. J Clin Microbiol 1999, 37:442-444.

29. Pestel-Caron M, Arbeit RD: Characterization of IS1245 for strain typing of Mycobacterium avium. J Clin Microbiol 1998, 36:1859-1863.

30. Tenover FC, Arbeit RD, Goering RV, Mickelsen PA, Murray BE, Persing DH, Swaminathan B: Interpreting chromosomal DNA restriction patterns produced by pulsed-field gel electrophoresis: criteria for bacterial strain typing. J Clin Microbiol 1995, 33:2233-2239

31. Arbeit RD, Slutsky A, Barber TW, Maslow JN, Niemczyk S, Falkinham JO, O'Connor GT, von Reyn CF: Genetic diversity among strains of Mycobacterium avium causing monoclonal and polyclonal bacteremia in patients with AIDS. J Infect Dis 1993, 167:1384-1390.

32. Oliveira RS, Sircili MP, Ueki SY, Telles MA, Schnabel B, Briones MR, Leao SC: PCR-restriction enzyme analysis of a bone marrow isolate from a human immunodeficiency virus-positive patient discloses polyclonal infection with two Mycobacterium avium strains. J Clin Microbiol 2000, 38:4643-4645.

33. Tirkkonen T, Pakarinen J, Moisander AM, Makinen J, Soini H, li-Vehmas T: High genetic relatedness among Mycobacterium avium strains isolated from pigs and humans revealed by comparative IS1245 RFLP analysis. Vet Microbiol 2007, 125:175-181.

34. Pate M, Zolnir-Dovc M, Krt B, Ocepek M: IS1245 RFLP-based genotyping study of Mycobacterium avium subsp. hominissuis isolates from pigs and humans. Comp Immunol Microbiol Infect Dis 2008, 31:537-550.

35. Matlova L, Dvorska L, Ayele WY, Bartos M, Amemori T, Pavlik I: Distribution of Mycobacterium avium complex isolates in tissue samples of pigs fed peat naturally contaminated with mycobacteria as a supplement. J Clin Microbiol 2005, 43:1261-1268.

36. Matlova L, Dvorska L, Palecek K, Maurenc L, Bartos M, Pavlik I: Impact of sawdust and wood shavings in bedding on pig tuberculous lesions in lymph nodes, and IS1245 RFLP analysis of Mycobacterium avium subsp. hominissuis of serotypes 6 and 8 isolated from pigs and environment. Vet Microbiol 2004, 102:227-236.

37. Alvarez J, Castellanos E, Romero B, Aranaz A, Bezos J, Rodriguez S, Mateos A, Dominguez $L$, de JL: Epidemiological investigation of a Mycobacterium avium subsp. hominissuis outbreak in swine. Epidemiol Infect 2011, 143-148.

doi:10.1186/1746-6148-7-63

Cite this article as: Agdestein et al:: Investigation of an outbreak of mycobacteriosis in pigs. BMC Veterinary Research 2011 7:63. 